\title{
()RIGIN.II
}

\section{FACTORES ASOCIADOS A LOS ACCIDENTES POR EXPOSICIÓN PERCUTÁNEA EN PERSONAL DE ENFERMERÍA DE UN HOSPITAL DE TERCER NIVEL}

\author{
M.a Teresa Gallardo López (1), Josefa Masa Calles (1), Rafael Fernández-Crehuet Navajas (1), \\ Jokin de Irala Estévez (2), Diego Martínez de la Concha (1) y Carmen Díaz Molina \\ (1) Servicio de Medicina Preventiva. Hospital Universitario Reina Sofía. Córdoba. \\ (2) Departamento de Medicina Preventiva y Salud Pública. Facultad de Medicina. Universidad de Córdoba.
}

\section{RESUMEN}

Fundamento: Los accidentes por exposición percutánea suponen aproximadamente un tercio de los accidentes laborales del personal sanitario hospitalario. Su importancia estriba en las enfermedades causadas por patógenos transmisibles por esta vía (hepatitis $B$, hepatitis $C$, infección VIH). Objetivos: describir los accidentes de este tipo notificados en un hospital de tercer nivel; identificar factores asociados a estos accidentes en personal de enfermería; construir un modelo predictivo del riesgo individual de accidentarse.

Métodos: Estudio descriptivo de una cohorte retrospectiva compuesta por todas las personas que notificaron un accidente entre el 1-1-93 y el 30-6-96. Estudio de casos y controles en el personal de enfermería durante el período 1-1-95 al 30-6-96, analizado mediante regresión logística múltiple.

Resultados: La incidencia acumulada de accidentes en un año fue de 0,078 para las/-os enfermeras/-os. En el 57,3\% de los casos estuvieron implicadas agujas de jeringas desechables o precargadas. La incidencia acumulada en un año fue mayor para las agujas de cateterismo intravenoso $(8,5$ por 100.000$)$. El riesgo de accidentabilidad, ajustado por variables confundentes, fue mayor para las/-os enfermeras/-os ( $O R=3,22$; I.C. $45 \%=1,96-5,27)$, para los trabajadores de la Unidad de Hemodiálisis $(\mathrm{OR}=35,21$; I.C.95\% $=3,74-331,16)$ y para aquéllos con contrato eventual ( $O R=4,50$; I.C.95\% $=2,24-9,04)$.

Conclusiones: Los accidentes por exposición percutánea en este hospital son más frecuentes entre el personal de enfermería y se producen, fundamentalmente, con algún tipo de aguja hueca. Se han identificado factores asociados a estos accidentes, lo que permite dirigir programas preventivos específicos sobre trabajadores con más riesgo. El modelo obtenido es válido para estimar el grado de accidentabilidad individual en los sujetos estudiados.

Palabras clave: accidentes, enfermería, factores de riesgo, prevención, agujas.

Correspondencia

M. Teresa Gallardo López

Departamento de Medicina Preventiva y Salud Pública.

Facultad de Medicina. Universidad de Córdoba

Avda Menéndez Pidal, s/n

14004 Córdoba

\section{ABSTRACT \\ Factors Associated with Accidents Resulting from Percutaneous Exposure in Nursing Staff at a Tertiary Level Hospital}

Background: Accidents resulting from percutaneous exposure account for approximatcly onc third of all accidents suffcred at work by health workers in hospitals. Their importance lies in the illnesses caused by pathogens that can be transmitted in this way (hepatitis B, hepatitis C, HIV virus). The aims are to describe accidents of this type notified in a tertiary level hospital, identify factors associated with these accidents in nursing staff and build a predictive model for the individual risk of having an accident.

Methods: A descriptive study of a retrospective cohort made up of all the people who notified having suffered an accident between 1-1-93 and 30-6-96. A study of cases and controls in nursing staff during the period 1-1-95 to 30-6-96, analysed through multiple logistical regression.

Results: The cumulative incidence of cases of accidents in one year was 0.078 for male and female nurses. In $57.3 \%$ of cases, disposable or pre-loaded syringes were involved. The cumulative incidence of cases in one year was greater for intravenous catheterisation $(8.5 \%$ per 100,000$)$. The risk of having an accident, adjusted on account of confusing variables, was greater for female and male nurses $(\mathrm{OR}=3.22$; I.C. $95 \%=1.96$ 5.27), for workers in the Haemodialysis Unit $(O R=35.21$; I.C. $.5 \%=3.74-331.16$ ) and for those employed on a temporary contract $(\mathrm{OR}=4.50 ;$ I.C. $.95 \%=2.24-9.04)$.

Conclusions: Accidents resulting from percutaneous exposure at this hospital are more frequent among nursing staff and are basically caused by any type of hollow needles. Factors associated with these accidents were identified, allowing specific prevention programmes to be targeted at those workers at greater risk. The model obtained is valid to estimate the degree of individual accident probability for the subjects studied.

Key words: Accidents. Nursing. Risk factors. Prevention. Needles. 


\section{INTRODUCCIÓN}

Los riesgos laborales de tipo biológico son los más frecuentes entre el personal sanitario hospitalario ${ }^{1}$. De ellos cabe destacar los accidentes por exposición percutánea, ya que suponen aproximadamente un tercio de los accidentes laborales de estos trabajadores ${ }^{2}$. Por categorías profesionales, la de enfermería presenta la mayor frecuencia ${ }^{1}$.

Los accidentes por exposición percutánea incluyen aquéllos que conllevan una penetración a través de la piel por una aguja u otro objeto punzante o cortante contaminado con sangre, con otro fluido que contenga sangre visible, con otros fluidos potencialmente infecciosos o con tejidos de un paciente. Dentro de los fluidos potencialmentc infecciosos se incluyen los líquidos cefalorraquídeo, sinovial, pleural, peritoneal, pericárdico y amniótico, así como el semen y las secreciones vaginales ${ }^{3}$.

De los, al menos, 20 patógenos diferentes que pueden transmitirse por esta vía ${ }^{4}$, los más importantes son el virus de la hepatitis $B$ (VHB), el virus de la inmunodeficiencia humana (VIH) y el virus de la hepatitis C (VHC), ya que las consecuencias de las enfermedades causadas por éstos son de gran trascendencia.

A partir de la vacunación sistemática frente a la hepatitis B (HB) de los trabajadores sanitarios la incidencia de ésta ha disminuido en gran medida, persistiendo el riesgo de infección por VIH y VHC, para los que no se dispone de vacuna por el momento.

Tras la inoculación accidental de sangre procedente de un paciente VIH positivo, el riesgo de seroconversión depende del tipo de exposición, siendo mayor para la percutánea $(0,23 \%)$ que para la mucocutánea $(0,08 \%)$, del volumen de sangre implicado y de la concentración de virus en ésta ${ }^{5,6}$.
Hasta octubre de 1995 se han registrado, en todo el mundo, 84 casos confirmados de infección por VIH en personal sanitario tras una exposición accidental en su trabajo, de los que 28 corresponden a países europeos (5 casos son españoles). De estos 28 , el $78,6 \%$ ocurrieron en profesionales de enfermería. Se han recogido otros 149 casos en los que no se ha podido confirmar su origen ocupacional'.

Para el caso de la hepatitis C (HC), las cifras de riesgo de seroconversión tras accidente por exposición percutánea, en la que el paciente fuente presente anticuerpos positivos para el VHC, varían según autores. Así, el riesgo se estima en un 3\% aproximadamente, sin embargo, si la sangre del paciente fuente es positiva para el RNA del VHC, el riesgo se eleva a un $10 \%$.

A pesar de las recomendaciones realizadas por organismos oficiales como los Centers for Disease Control (CDC), la Occupational Safety and Health Administration (OSHA), y la Food and Drug Administration (FDA $)^{9}$, los trabajadores sanitarios siguen accidentándose y realizando su tarea no siempre de la forma más segura. Una de las razones principales para que ésto ocurra es que cada hospital tiene sus propios factores de riesgo, que deben ser identificados para poder implantar programas adecuados de prevención $^{10}$.

Por tanto, los objetivos principales de este estudio son los siguientes: identificar factores asociados a los accidentes por exposición percutánea notificados por el personal de enfermería de un hospital de tercer nivel, y construir un modelo estadístico que permita estimar el riesgo individual de accidentarse. Como objetivo secundario se propone una descripción de la frecuencia y características de estos accidentes.

\section{MATERIAL Y MÉTODOS}

La investigación se llevó a cabo en el Hospital Universitario Reina Sofía (HURS) de 
Córdoba. Dicho hospital consta de 1.510 camas y en él trabajan 660 médicos, 237 facultativos residentes, 1.332 enfermeras, 953 auxiliares de enfermería y 124 técnicos. Sc realizaron dos discños distintos: un cstudio descriptivo de una cohorte retrospectiva y un estudio de casos y controles.

Así, para el primer diseño, el sujeto de estudio fue el personal sanitario del HURS perteneciente a las siguientes categorías: médicos, médicos internos residentes (MIR), enfermeras/-os (incluidas matronas), auxiliares de enfermería y técnicos de laboratorio. Se excluyeron aquellos trabajadores que, aún pcrteneciendo a una de estas categorías, no se encontraban expuestos. Éste fue el caso del personal adscrito a los servicios de dirección, subdirección, admisión, documentación clínica, docencia e investigación e información al usuario (este criterio de exclusión se mantuvo para el estudio de casos y controles). El período de estudio considerado fue el comprendido entre el 1-1-93 y el 30-6-96 (3,5 años) y los accidentes por exposición percutánea se obtuvieron de los notificados al Servicio de Medicina Preventiva (SMP) de dicho hospital durante el citado período de tiempo.

Se hizo una selección de variables de interés, quedando incluidas en el estudio el sexo, la edad, la categoría profesional, el área de trabajo, el tipo de objeto implicado en el accidente, cómo ocurrió el accidente, la indicación de seguimiento serológico y la cumplimentación del seguimiento por parte del trabajador. Estas variables son parte del total de las propuestas en uno de los registros cxistentes de trabajadores sanitarios expuestos a contactos con sangre $\mathbf{u}$ otros productos procedentes de pacientes, como es el EPINet (Exposure Prevention Information Network) ${ }^{11}$.
En el análisis se realizó la distribución de frecuencias de las variables cualitativas consideradas. Para la edad, única variable cuantitativa, se calcularon medidas de tendencia central y de dispersión; posteriormente se trató como variable cualitativa con tres categorías que coinciden con los siguientes grupos de edad: 20-30 años, 3150 años y 51-66 años. Los límites de dichas categorías agrupan personas con características similares en cuanto a habilidades y destreza adquirida en el desarrollo de su actividad profesional.

Como aproximación al cálculo de la incidencia acumulada (IA) global y específica por sexo, edad, categoría profesional y área de trabajo, se utilizó la siguiente fórmula:

$\frac{\mathrm{N} .{ }^{\circ} \text { de trabajadores accidentados (global o específico) }}{\mathrm{N} .^{\circ} \text { de trabajadores expuestos (global oespecífico) }}$

Los trabajadores con más de un accidente notificado en un año, solamente fueron contabilizados una vez en el numerador ese año. Para la obtención de los denominadores se utilizó la base de datos del personal de fecha 30-6-95, por ser la disponible en el momento de llevar a cabo el estudio; por otra parte, la movilidad del personal en un hospital con gran número de trabajadores, como es el HURS, es despreciable.

El resultado informa del riesgo que tiene un trabajador de sufrir un accidente en el período de 3,5 años estudiado. Para aproximarnos al valor de dicho riesgo referido a un año se dividió este resultado entre 3,5.

Para el cálculo de la IA específica por tipo de objeto se utilizaron datos referentes al año 1.995, ya que eran los disponibles en el momento de la realización de esta investigación. El cálculo de esta IA se realizó según la siguiente expresión:

$\frac{\mathrm{N} .^{\circ} \text { de accidentes atribuido a un tipo de objeto en el período considerado }}{\mathrm{N} .^{\circ} \text { de objetos de ese tipo consumidos en el período considerado }} \times 100.00$ 
La cifra así obtenida informa de la probabilidad de sufrir un accidente al utilizar un determinado objeto en un año (expresado por 100.000 de esos objetos consumidos en un año).

Una vez calculada la IA se obtuvo el riesgo relativo (RR) para cada categoría dentro de cada variable, tomando como referencia la categoría de la variable en cuestión con una menor IA.

Por otro lado, se llevó a cabo un estudio de casos y controles. La población de estudio fue el personal sanitario del HURS perteneciente a las categorías de enfermería y auxiliar de enfermería, por ser el colectivo con mayor frecuencia de accidentes por exposición percutánea ${ }^{1}$.

El tamaño muestral se determinó a partir de las tablas de Schlesselman para un nivel de confianza del $95 \%(\alpha=0,05)$, una potencia del $80 \%(\beta=0,20)$ y una Odds Ratio (OR) mínima a detectar de 2 . Se consideró una proporción de expuestos en el grupo control del $15 \%$ para aquella variable independiente de la que se esperaba una menor proporción (haber notificado al menos un accidente por exposición percutánea en los 4 años previos al período de estudio) y se tomaron dos controles por caso. Para cumplir estos requisitos el tamaño muestral necesario fue de 492 (164 casos y 328 controles) ${ }^{12}$.

Se definió como caso aquel trabajador perteneciente a la categoría de enfermería o auxiliar de enfermería del HURS, que hubiera notificado al menos un accidente por exposición percutánea al SMP en el período comprendido entre el 1-1-95 y el 30-6-96. Se utilizaron todos los casos de dicho período. Se consideró control elegible aquel trabajador perteneciente a dichas categorías profesionales que, habiendo trabajado en este hospital en el período de estudio, no hubiera notificado ningún accidente de este tipo al SMP. La elección de los controles se hizo de forma aleatoria de una base de datos construida con todos los controles elegibles.
Las variables independientes consideradas fueron: sexo, edad, categoría profesional, área de trabajo, antigüedad en el hospital, tipo de contrato, turno de trabajo y haber notificado al menos un accidente por exposición percutánea en los 4 años previos al período de estudio.

Las variables antigüedad cn el hospital y tipo de contrato se incluyeron para medir indirectamente la experiencia profesional. Como medida indirecta de la carga de trabajo se incluyó la variable turno de trabajo. El hecho de haber notificado algún accidente en los 4 años previos al período de estudio se tuvo en cuenta para ayudar a valorar la existencia de alguna característica intrínseca de la persona que la haga accidentarse más.

El análisis se llevó a cabo mediante regresión logística múltiple, previa descripción de la muestra. Para la construcción del modelo se efectuó una primera elección de variables realizando una selección manual de tipo metódica (purposeful selection) de modelos univariantes, con criterios conservadores de selección ( $p$ alrededor de 0,25 ). Con las variables que alcanzaron este nivel de significación se ajustó un modelo multivariante, que fue depurado posteriormente eliminando variables con la ayuda del test de la razón de verosimilitud (estadístico $G$ ). Se llegó al modelo final tras realizar las pruebas pertinentes para identificar posibles interacciones (cambio significativo del logaritmo de la verosimilitud al introducir la interacción) o factores de confusión (método de la proporción de cambio de los coeficientes del modelo). En este último caso se consideró un cambio del coeficiente superior al $15 \%$ para incluir a la variable en cuestión como factor de confusión. También se valoró la bondad de ajuste del modelo final con la prueba de la $\mathrm{X}^{2}$ de Pearson ${ }^{13}$.

Una vez construido este modelo, se procedió a calcular la probabilidad de que una persona se accidente conociendo sus características respecto a las variables indepen- 
dientes incluidas en él. La fórmula a aplicar fue la siguiente:

$$
\pi(x)=\frac{e^{g(x)}}{1+e^{g(x)}}
$$

donde $g(x)=\beta_{0}+\beta_{1} x_{1}+\ldots+\beta_{p} x_{p .}$ Las $\beta_{i}$ representan los coeficientes del modelo final $\mathrm{y}$ las $\mathrm{x}_{\mathrm{i}}$ las variables incluidas en dicho modelo. En los estudios de casos y controles, el valor adecuado de la constante $\left(\beta_{0)}\right.$ para hallar probabilidades de presentar la respuesta de interés depende de la fracción de muestreo y su valor se obtiene despejando de la siguiente fórmula:

$$
\beta_{0}^{\prime}=\ln \frac{P_{1}}{P_{2}}+\beta_{0}
$$

donde $\beta_{0}$ ' es la constante del modelo obtenida sin tener en cuenta la fracción de muestreo; $\mathrm{P}_{1}$ es la proporción de casos de la muestra respecto del total de casos ocurridos en el período de estudio, en esta ocasión su valor será 1 puesto que se han tomado todos los casos; $y$ $\mathrm{P}_{2}$ es la proporción de controles de la muestra respecto del total de controles elegibles ${ }^{13}$, el $16,2 \%$ en este estudio.

Para todo este análisis se utilizaron los programas dBASE III PLUS versión 1.0, EPI-INFO versión 6.02 y STATISTIX versión 4.1 .

\section{RESULTADOS}

Estudio descriptivo de una cohorte retrospectiva:

De los 569 accidentes por exposición percutánea notificados al SMP durante el período de estudio, 174 ocurrieron en el año 93, 160 en el 94, 158 en el 95 y 77 en el primer semestre del 96 . La IA por persona en un año fue de 0,053 para el año 93 (I.C. $95 \%=0,046-0,061) ; 0,049$ para el 94 (I.C. $95 \%=0,042-0,057)$ y 0,048 para el 95 (I.C. $.95 \%=0,04-0,056)$.
Tabla 1

Distribución de las variables consideradas en el estudio

\begin{tabular}{|c|c|c|}
\hline Variable & $n$ & $\%$ \\
\hline \multicolumn{3}{|l|}{ SEXO: } \\
\hline - Hombres & 127 & 22,3 \\
\hline - Mujeres & 442 & 77,7 \\
\hline Total & 569 & 100,0 \\
\hline \multicolumn{3}{|l|}{ EDAD: } \\
\hline$-20-30$ años & 140 & 24,6 \\
\hline$-31-50$ años & 375 & 65,9 \\
\hline$-51-66$ años & 54 & 9,5 \\
\hline Total & 569 & 100,0 \\
\hline \multicolumn{3}{|l|}{ CATEGORÍA PROFESIONAL: } \\
\hline - Mćdico & 55 & 9,6 \\
\hline - Médico interno residente & 44 & 7,7 \\
\hline - Enfermeras/-os & 360 & 63,3 \\
\hline - Auxiliar de enfermería & 99 & 17,4 \\
\hline - Técnico de laboratorio & 11 & 1,9 \\
\hline Total & 569 & 100,0 \\
\hline \multicolumn{3}{|l|}{ ÁREA DE TRABAJO: } \\
\hline — Médicas & 144 & 25,3 \\
\hline - Quirúrgicas & 237 & 41,6 \\
\hline - Unidad de Hemodiálisis & 33 & 5,8 \\
\hline -- Urgencias & 56 & 9,8 \\
\hline - Unidad de cuidados intensivos & 42 & 7,4 \\
\hline - Laboratorios & 42 & 7,4 \\
\hline - Otros & 15 & 2,7 \\
\hline Total & 569 & 100,0 \\
\hline \multicolumn{3}{|l|}{ OBJETO IMPLICADO EN EL ACCIDENTE: } \\
\hline $\begin{array}{l}\text { - Aguja de jeringas desechables o pre- } \\
\text { cargadas }\end{array}$ & 326 & 57,3 \\
\hline — Aguja de jeringas de gasometría & 21 & 3,7 \\
\hline - Aguja de cateterismo intravenoso & 56 & 9.8 \\
\hline — Aguja de sutura & 73 & 12,8 \\
\hline - Bisturí & 47 & 8,2 \\
\hline - Lanceta & 9 & 1,6 \\
\hline _- Otras agujas & 14 & 2,5 \\
\hline - Otro instrumental quirúrgico & 23 & 4,1 \\
\hline Total & 569 & 100,0 \\
\hline \multicolumn{3}{|l|}{ CÓMO HA OCURRIDO: } \\
\hline - Durante el uso del material & 235 & 41,3 \\
\hline - Recogiendo material tras su uso & 158 & 27,7 \\
\hline - Preparando material reutilizable & 15 & 2,7 \\
\hline - Reencapuchando & 54 & 9,5 \\
\hline - Retirando la aguja de material resistente & 8 & 1,4 \\
\hline - Otros usos antes del desecho & 48 & 8.4 \\
\hline — Dejando objeto en contenedor & 12 & 2,1 \\
\hline - Con objeto sobresaliente en contenedor & 24 & 4,2 \\
\hline $\begin{array}{l}\text { - Con objeto sobresaliente en bolsa de } \\
\text { basura }\end{array}$ & 5 & 0,9 \\
\hline - Otros & 10 & 1,8 \\
\hline Total & 569 & 100,0 \\
\hline
\end{tabular}
descriptivo 
Tabla 1 (continuación)

\begin{tabular}{|lrr|}
\hline Variable & $n$ & $\%$ \\
\hline INDICACIÓN DE SEGUIMIENTO & & \\
SEROLÓGICO: & 189 & 33,2 \\
- Sí & 380 & 66,8 \\
- No & 569 & 100,0 \\
Total & & \\
CUMPLIMENTACIÓN SEGUIMIENTO & & \\
VIH: & 42 & 7,4 \\
- Completa (1 año) & 13 & 2,3 \\
- Hasta $6 .^{\circ}$ mes & 12 & 2,1 \\
- Hasta 3. ${ }^{\circ}$ mes & 25 & 4,4 \\
- No cumplimentación & 24 & 4,2 \\
- Continúa en seguimiento & 453 & 79,6 \\
- No procede & 569 & 100,0 \\
Total & & \\
CUMPLIMENTACIÓN SEGUIMIENTO & & \\
VHC: & 55 & 9,6 \\
- Completa (l año) & 22 & 3,9 \\
- Hasta 6. ${ }^{\circ}$ mes & 15 & 2,6 \\
- Hasta 3. ${ }^{\circ}$ mes & 38 & 6,7 \\
- No cumplimentación & 43 & 7,6 \\
— Continúa en seguimiento & 396 & 69,6 \\
- No procede & 569 & 100,0 \\
\hline Total & & \\
\hline
\end{tabular}

VIH: virus de la inmunodeficiencia humana.

VHC: virus de la hepatitis $C$

En la tabla 1 se recoge la distribución de frecuencias de las variables de estudio y en la tabla 2 la IA y RR en un año específicos por sexo, edad, categoría profesional, área de trabajo y tipo de objeto implicado en el accidente. De ellas destacan los hallazgos que se citan a continuación.

El 77,7\% de estos accidentes sucedió en mujeres. La IA fue mayor para éstas con un valor de 0,052 por mujer/año, mientras que para los hombres fue de 0,038 . Así, el riesgo de accidentabilidad fue 1,37 veces superior para las mujeres en comparación con los hombres.

La media de edad de los trabajadores accidentados fue de 37,6 años, con una desviación estándar (DE) de 9,2 años. Aproximadamente dos tercios de los accidentes ocurrieron en el grupo de edad de 31-50 años. Sin embargo, fue el grupo de 20-30 años el que presentó una mayor IA por persona en un año $(0,074)$, así
Tabla 2

Incidencia acumulada en un año y riesgo relativo específicos por sexo, edad, categoría profesional, área de trabajo y tipo de objeto implicado en el accidente

\begin{tabular}{|c|c|c|}
\hline & $I A$ & $R R(I . C .95 \%)$ \\
\hline \multicolumn{3}{|l|}{ SEXO: } \\
\hline - Hombres & 0,038 & 1,00 \\
\hline - Mujeres & 0,052 & $1,37(1,14-1,64)$ \\
\hline \multicolumn{3}{|l|}{ EDAD: } \\
\hline$-20-30$ años & 0,074 & $2,55(1,89-3,38)$ \\
\hline$-31-50$ años & 0,047 & $1,62(1,23-2,10)$ \\
\hline$-51-66$ años & 0,029 & 1,00 \\
\hline \multicolumn{3}{|c|}{ CATEGORÍA PROFESIONAL: } \\
\hline - Médico & 0,024 & 1,00 \\
\hline - Médico interno residente & 0.054 & $2,25(1,54-3,22)$ \\
\hline - Enfermeras/-os & 0,078 & $3,25(2,44-4,17)$ \\
\hline - Auxiliar de enfermería & 0,038 & $1,25(0,89-1,67)$ \\
\hline — Técnico de laboratorio & 0,025 & $1,04(0,56-1,93)$ \\
\hline \multicolumn{3}{|l|}{ ÁREA DE TRABAJO: } \\
\hline - Médicas & 0,031 & $1,48(0,90-2,50)$ \\
\hline — Quirírgicas & 0,056 & $2,67(1,62-4,42)$ \\
\hline - Hemodiálisis & 0,147 & $7,00(4,08-12,06)$ \\
\hline - Urgencias & 0,095 & $4,52(2,65-7,67)$ \\
\hline \multicolumn{3}{|l|}{$\begin{array}{l}\text { - Unidad de cuidados inten- } \\
\text { sivos }\end{array}$} \\
\hline - Laboratorios & 0,050 & $2,38(1,35-4,13)$ \\
\hline \multirow[t]{2}{*}{ - Otros* } & 0,021 & 1,00 \\
\hline & $\begin{array}{l}\text { IA por } \\
100.000\end{array}$ & $R R$ \\
\hline \multicolumn{3}{|c|}{ OBJETO IMPLICADO EN EL ACCIDENTE**: } \\
\hline $\begin{array}{l}\text { - Aguja de jeringas dese- } \\
\text { chables o precargadas }\end{array}$ & 4,2 & $1,31(0,68-1,65)$ \\
\hline $\begin{array}{l}\text { - Aguja de jeringas de gaso- } \\
\text { metría }\end{array}$ & 3,2 & 1,00 \\
\hline $\begin{array}{l}\text { - Aguja de cateterismo in- } \\
\text { travenoso }\end{array}$ & 8,5 & $2,66(1,64-4,46)$ \\
\hline — Aguja de sutura & - & - \\
\hline - Bisturí & - & - \\
\hline - Lanceta & - & -- \\
\hline - Otras agujas & - & - \\
\hline $\begin{array}{l}\text { - Otro instrumental quirú- } \\
\text { gico }\end{array}$ & - & - \\
\hline
\end{tabular}

IA: Incidencia acumulada.

RR (I.C.95\%): Riesgo relativo e intervalo de confianza al $95 \%$.

* Radiodiagnóstico, Esterilización y Odontología.

** Sólo pudo calcularse para agujas huecas, por falta de disponibilidad de los denominadores del resto de los objetos.

como un mayor RR $(2,55)$ tomando como referencia el grupo de 51-66 años.

Según la categoría profesional, se observó que más de la mitad de los accidentes se 
produjcron en enfermeras/-os $(63.3 \%)$. Así mismo, es esta categoría la que presentó el mayor riesgo de accidentabilidad por persona con una IA de 0,078 en un año, mientras que el menor resultó ser para los médicos $(0,024)$. Así, comparando ambas categorías, el riesgo fue 3,25 veces superior para la de enfermeras/-os.

La mayoría de los accidentes $(41,6 \%)$ tuvieron lugar en áreas quirúrgicas. La IA por persona en un año específica por área de trabajo fue más elevada para la unidad de hemodiálisis $(0,147)$, mientras que urgencias ocupó el segundo lugar $(0,095)$ seguida de la UCI $(0,068)$. Destaca, en este mismo orden, los RR de 7,00, 4,52 y 3,24 respectivamente, tomando de referencia el área denominada Otros (radiodiagnóstico, esterilización y odontología).

El objeto más frecuentemente involucrado en los accidentes fue la aguja de jeringas desechables o precargadas, que ocasionó el $57,3 \%$ de dichos accidentes. Sin embargo, la IA anual más alta fue para las agujas de cateterismo intravenoso (IV) $(8,5$ por 100.000 ), mientras que las agujas de gasometría presentaron la IA más baja (3,2 por 100.000). Comparando ambas, el riesgo de accidentarse fue 2,66 veces mayor para las agujas de cateterismo (IV).

Teniendo en cuenta la actividad que sc estaba realizando en el momento del accidente, resultó que más de dos tercios de éstos sucedieron, bien durante el uso del material $(41,3 \%)$ o bien durante su recogida tras acabar el procedimiento $(27,7 \%)$. Por otro lado, el $9,5 \%$ de los accidentes se produjeron durante el reencapuchado de una aguja usada, mientras que actividades relacionadas con otro uso del material antes del desecho como dejarlo en la cama, mesa, suelo $\mathrm{u}$ otro lugar inapropiado se relacionó con el $8,4 \%$ de los mismos.

El $33,2 \%$ de los accidentes precisaron seguimiento serológico, bien por presentar el paciente fuente anticuerpos positivos para el VHC y/o VIH, bien por desconocerse la serología del mismo con respecto a dichos anticuerpos. Aproximadamente un tercio de los trabajadores a los que se les indicó seguimiento para el VIH lo completó, siendo similar el porcentaje para el caso del VHC.

Es importante destacar que no hubo ninguna seroconversión entre los trabajadores accidentados durante el período de estudio para el VHC ni para el VIH.

\section{Estudio de casos y controles:}

La mayor proporción de casos y de controles fueron mujeres $(90,9 \%$ y $87,2 \%$ respectivamente). La media de edad fue de 39,5 años con una DE de 8,9 años para los casos y de 41,2 años con una DE de 9,2 años para los controles. El 57,0\% de estos últimos eran enfermeras/-os, mientras que este porcentaje fue del 81,7 para los casos. La distribución del resto de variables de estudio se resume en la tabla 3.

El modelo que predice el riesgo de accidentarse se muestra en la tabla 4. En él destaca el mayor riesgo de accidentabilidad de las enfermeras/-os, de los trabajadores de la unidad de hemodiálisis, urgencias y laboratorios, del personal con un tipo de contrato eventual, así como de aquéllos que habían sufrido uno de estos accidentes en los 4 años previos al período de estudio. El sexo se incluyó en el modelo por actuar como factor de confusión para algunas de las variables dummies de la variable área de trabajo.

En dicho modelo no existió evidencia de falta de ajuste $\left(x^{2}=45,97 ; p=0,2057\right.$ con 39 grados de libertad).

Se estimó la probabilidad individual de accidentarse utilizando el modelo final, oscilando dicha probabilidad entre un mínimo de 0,0026 y un máximo de 0,4746 (media = $0,0785, \mathrm{DE}=0,0916$ ). En la Tabla 5 se presenta la distribución de frecuencias de los accidentes por exposición percutánea en cuatro grupos de riesgo confeccionados a partir de los tres cuartiles de la variable probabilidad indi- 
Tabla 3

Distribución de las variables consideradas en el estudio de casos y controles

\begin{tabular}{|c|c|c|c|c|}
\hline \multirow[t]{2}{*}{ Variables cualitativas } & \multicolumn{2}{|c|}{ Casos $(n=164)$} & \multicolumn{2}{|c|}{ Controles $(n=328)$} \\
\hline & $n$ & $\%$ & $n$ & $\%$ \\
\hline \multicolumn{5}{|l|}{ SEXO: } \\
\hline - Hombres & 15 & 9,1 & 42 & 12,8 \\
\hline - Mujeres & 149 & 90,9 & 286 & 87,2 \\
\hline \multicolumn{5}{|l|}{ CATEGORÍA PROFESIONAL: } \\
\hline - Auxiliar de enfermería & 30 & 18,3 & 141 & 43,0 \\
\hline - Enfermeras/-os & 134 & 81,7 & 187 & 57,0 \\
\hline \multicolumn{5}{|l|}{ ÁREA DE TRABAJO: } \\
\hline - Médicas & 44 & 26,8 & 124 & 37,8 \\
\hline - Quirúrgicas & 64 & 39,0 & 124 & 37,8 \\
\hline - Unidad de Hemodiálisis & 15 & 9,1 & 6 & 1,8 \\
\hline - Urgencias & 15 & 9,1 & 20 & 6,1 \\
\hline - Unidad de cuidados intensivos & 13 & 7,9 & 22 & 6,7 \\
\hline - Laboratorios & 12 & 7,3 & 12 & 3,7 \\
\hline - Otros* & 1 & 0,6 & 20 & 6,1 \\
\hline \multicolumn{5}{|l|}{ TIPO DE CONTRATO: } \\
\hline- Fijo & 137 & 83,5 & 311 & 94,8 \\
\hline - Eventual & 27 & 16,5 & 17 & 5,2 \\
\hline \multicolumn{5}{|l|}{ TURNO DE TRABAJO: } \\
\hline - Diurno & 48 & 29,3 & 101 & 30,8 \\
\hline - Rotatorio & 111 & 67,7 & 199 & 60,7 \\
\hline - Noches alternas & 5 & 3,0 & 28 & 8,5 \\
\hline \multicolumn{5}{|l|}{ ACCIDENTE PREVIO: } \\
\hline- No & 117 & 71,3 & 275 & 83,8 \\
\hline — Sí & 47 & 28,7 & 53 & 16,2 \\
\hline Variables cuantitativas (años) & Media & D.E. & Media & D.E. \\
\hline EDAD & 39,5 & 8,9 & 41,2 & 9,2 \\
\hline ANTIGÜEDAD EN EL HOSPITAL & 10,6 & 7,2 & 11,0 & 6,8 \\
\hline
\end{tabular}

DE: Desviación estándar. * Radiodiagnóstico, Esterilización y Odontología.

vidual de accidentarse, se observa que la IA es mayor a medida que aumenta la probabilidad estimada por el modelo.

\section{DISCUSIÓN}

Al igual que se recoge en otros estudios ${ }^{2}$, los accidentes por exposición percutánea en el HURS representan aproximadamente un tercio del total de accidentcs laborales registrados. En cuanto al riesgo de accidentabilidad por persona en un año, también es comparable al de otros hospitales de España ${ }^{1}$.
El hecho de que el grupo de edad más joven (20-30 años) haya presentado un mayor riesgo de accidentarse puede estar relacionado con una mayor inexperiencia de estas personas, ya que, por lo general, suelen estar comenzando su carrera profesional.

Si tenemos en cuenta la categoría profesional, son las/-os enfermeras/-os las/-os que presentan un mayor riesgo de accidentabilidad. Ésto concuerda con lo detectado por otros autores ${ }^{1,2,14}$, si bien hay que ser cautelosos a la hora de comparar cifras con estudios americanos, ya que existen dife- 
Tabla 4

Variables asociadas a los accidentes por exposición percutánea en el Hospital Universitario Reina Sofia

\begin{tabular}{|c|c|c|c|c|c|}
\hline Variables & Coeficientes & $p$ de Wald & $\begin{array}{c}\text { Límite } \\
\text { inferior al } \\
95 \%\end{array}$ & Odds Ratio & $\begin{array}{l}\text { Límite superior } \\
\text { al } 95 \%\end{array}$ \\
\hline CONSTANTE & $-4,12241$ & 0,0001 & - & - & - \\
\hline \multicolumn{6}{|l|}{ CATEGORÍA PROFESIONAL } \\
\hline $\begin{array}{l}\text { Enfermeras'-os } \\
\text { (referencia = Auxiliar de enfermería) }\end{array}$ & 1,16801 & 0,0000 & 1,96 & 3,22 & 5,27 \\
\hline \multicolumn{6}{|l|}{ ÁREA DE TRABAJO } \\
\hline Médicas & 1,47111 & 0,1611 & 0,56 & 4,35 & 34,08 \\
\hline Quirúrgicas & 1,88685 & 0,0708 & 0,85 & 6,60 & 51,12 \\
\hline Unidad de Hemodiálisis & 3,56121 & 0,0018 & 3,74 & 35,21 & 331,16 \\
\hline Urgencias & 2,25525 & 0,0399 & 1,11 & 9,54 & 81,94 \\
\hline Unidad de cuidados intensivos & 1,88801 & 0,0847 & 0,77 & 6,61 & 56,52 \\
\hline $\begin{array}{l}\text { Laboratorios } \\
\text { (referencia }=\text { Otros*) }\end{array}$ & 2,30703 & 0,0378 & 1,14 & 10,04 & 88,59 \\
\hline \multicolumn{6}{|l|}{ TIPO DE CONTRATO } \\
\hline $\begin{array}{l}\text { Eventual } \\
(\text { referencia }=\text { Fijo })\end{array}$ & 1,50405 & 0,0000 & 2,24 & 4,50 & 9,04 \\
\hline ACCIDENTE PREVIO & & & & & \\
\hline $\begin{array}{l}\text { Sí } \\
\text { (referencia = No) }\end{array}$ & 0,55073 & 0,0281 & 1,06 & 1,73 & 2,84 \\
\hline SEXO & & & & & \\
\hline $\begin{array}{l}\text { Mujeres } \\
\text { (referencia = Hombres) }\end{array}$ & 0,56022 & 0,1130 & 0,88 & 1,75 & 3,50 \\
\hline
\end{tabular}

Variable dependiente: accidente por exposición percutánea. Estadístico $\mathrm{G}=83,62 ; \mathrm{p}<0,0001$ con 10 grados de libertad. * Radiodiagnóstico, Esterilización y Odontología.

rencias en cuanto a las actividades realizadas por cada categoría profesional. Por ejemplo, los trabajadores de laboratorio en Estados Unidos (clinical laboratory wor-

Tabla 5

Distribución de los accidentes por exposición percutánea en cuatro grupos de riesgo

\begin{tabular}{|lrc|}
\hline $\begin{array}{c}\text { Grupos de riesgo (probabilidad } \\
\text { de accidentarse) }\end{array}$ & $n$ & $\begin{array}{c}\text { Incidencia } \\
\text { acumulada* }\end{array}$ \\
\hline Por debajo del $1 .^{\text {er }}$ cuartil & 104 & 2,9 \\
Entre el $1 .^{\text {er }}$ y $2 .^{\circ}$ cuartil & 93 & 3,2 \\
Entre el $2 .^{\circ}$ y $3 .^{\text {er }}$ cuartil & 91 & 9,9 \\
Por encima del 3. ${ }^{\text {er }}$ cuartil & 67 & 17,9 \\
\hline
\end{tabular}

n: Número de sujetos en cada grupo de riesgo.

* Incidencia acumulada de accidentes por exposición percutánea por 100 personas en el período de estudio ( 1,5 años). kers) son los encargados de realizar las extracciones sanguíneas, mientras que en la mayoría de los hospitales europeos son las/-os enfermeras/-os las/-os que llevan a cabo esta tarea ${ }^{5}$. En este estudio debemos tener presente la posibilidad de un sesgo de selección debido a la existencia de infranotificación de este tipo de accidentes, sobre todo entre los médicos. Las cifras de dicha infranotificación varían entre el $10 \%$ y el $68 \%$ según el estamento profesional o el área de trabajo estudiada ${ }^{15-18}$. No obstante, también existe documentación acerca de una tendencia por parte del personal sanitario, fundamentalmente de los médicos, a seleccionar los accidentes que notifican, declarando más aquéllos en los que el paciente tenía una enfermedad infecciosa po- 
tencialmente transmisible conocida, o bien el paciente fuente era desconocido ${ }^{3}$. Es de este tipo de accidentes de mayor riesgo del que más nos interesa recabar información para incidir sobre ellos; y así, de haber sesgo de selección, el presente estudio será probablemente más representativo de este tipo de sujetos y accidentes.

El mayor riesgo de accidentabilidad detectado para los trabajadores de la unidad de hemodiálisis puede relacionarse con el elevado número de procedimientos realizados en esta unidad que implican la utilización de algún tipo de aguja. Además, este dato es importante por el alto porcentaje de hepatitis $\mathrm{C}$ existente entre los enfermos dializados.

La carga asistencial del servicio de urgencias (el HURS es hospital de referencia para las provincias de Córdoba y Jaén), así como el estrés con que se suele trabajar en este área, puede ayudar a comprender el que sea una de las zonas con mayor riesgo de accidentabilidad. Por otro lado, en este área cl mayor número de accidentes corresponde a enfermeras/-os y auxiliares de enfermería, que es el personal que manipula un mayor número de objetos punzantes y cortantes.

Por tanto, de lo expuesto hasta el momento se deduce la relevancia de profundizar en el estudio del riesgo de los trabajadores de las categorías de enfermería y auxiliar de enfermería.

Al igual que hicieran G. Ippolito et al, en uno de sus estudios ${ }^{19}$, las agujas de jeringas desechables y las de jeringas precargadas se han incluido en el mismo grupo en este trabajo, ya que ambos tipos requieren poca manipulación en cuanto a desensamblaje.

El riesgo de accidentabilidad atribuido a cada tipo de objeto, de los que se conocía el consumo, fue similar al encontrado en otros estudios $^{4,19}$, coincidiendo en el hecho de que aquellos objetos que requieren desensamblaje implican un riesgo mayor, como es el caso de las agujas de cateterismo IV, mientras que el riesgo disminuye para los objetos que requieren menos manipulación, como las agujas de jeringas desechables y de las precargadas.

Es importante conocer el tipo de objeto implicado en los accidentes para poder incidir sobre aquéllos que más riesgo conlleven. La mejor solución sería reducir al máximo la utilización de objetos con aguja buscando otros métodos alternativos, o diseñar objetos más seguros para el trabajador. Pero esto hace necesaria la realización de estudios acerca del coste que ello supondría. Hay autores que han determinado el coste de estos accidentes atribuido a un tipo de objeto, el coste del objeto en cuestión y el porcentaje que representa el primero con respecto al segundo, llegando a la conclusión de que se disminuirían los accidentes relacionados con algunos tipos concretos de objetos y se ahorraría, desde el punto de vista económico, si se introdujeran otros más seguros ${ }^{20}$.

La probabilidad de transmisión de una enfermedad infecciosa como consecuencia de un accidente por exposición percutánea, es mayor en el caso de las agujas huecas y, dentro de éstas, es mayor para aquéllas de más calibre ya que pueden contener mayor cantidad de sangre ${ }^{4}$. Si a esto unimos el que, dentro de las categorías de enfermería y de auxiliar de enfermería, el objeto involucrado en la mayoría de sus accidentes fue algún tipo de aguja hueca, nos hace de nuevo pensar en este grupo a la hora de intervenir.

Al igual que en otros estudios ${ }^{10}$, las actividades más frecuentemente realizadas en el momento del accidente fueron las relacionadas con el uso del material o su manipulación antes del desecho. En este grupo de accidentes nos encontramos con factores sobre los que no sería tan fácil actuar, como son los derivados del paciente (por ejemplo, que éste se encuentre agitado y se mueva provocando el accidente). Sin embargo, existen otras circunstancias sobre las que se podría incidir, éste es el caso de los accidentes en los que se ven involucradas agujas que se encontraban en la mesa, suelo, cama u 
otro lugar inapropiado, que ocuparon el cuarto lugar de frecuencia en este cstudio.

El reencapuchado de una aguja usada ocasionó un porcentaje menor de accidentes en nuestro centro que el detectado en otros hospitales $^{1,4}$. Ésto podría atribuirse a una disminución de la realización de esta práctica, ya que cada vez que un trabajador acude a notificar uno de estos accidentes al SMP, se hace especial hincapić en la necesidad de evitar dicha maniobra, y a su vez esta persona puede influir sobre sus compañeros de trabajo. No obstante, en el caso del reencapuchado habría que indagar sobre las causas que llevaron al trabajador a realizarlo, ya que no siempre es debido a una mala práctica si no que pueden influir otros factores como la ausencia de un contenedor apropiado en el lugar preciso.

El seguimiento serológico, tanto para el VIH como para el VHC, sólo se completó en un tercio de los casos en los que hubo indicación. Ésto puede ser debido a relajación por parte del trabajador, una vez que le ha tranquilizado el hecho de que la primera determinación serológica haya sido negativa, o bien a que su interés era únicamente el que el accidente quedara registrado en el SMP.

Entre los accidentes notificados en el período de estudio no hubo ningún caso de seroconversión para el VIH ni para el VHC. Sin embargo el riesgo existe, y así está documentado en la bibliografía ${ }^{5,8,21}$, por lo que no se puede bajar la guardia a este respecto. Uno de los motivos que puede explicar el que no se haya detectado ningún caso de seroconversión en el período de estudio, podría ser un número insuficiente de accidentes con una fuente positiva. Por ejemplo, para el caso del VIH se necesitan 1.000 accidentes en los que el paciente fuente tuviera Ac VIH positivos para detectar $2 \mathrm{ca}$ sos de seroconversión, mientras que en este estudio el número de dichos accidentes es de 32 .
Con lo expuesto hasta el momento ya se han detectado algunos aspectos relevantes del problema de estudio para profundizar sobre ellos, y como primer paso se realizó una investigación con un diseño de casos y controles.

El primer hallazgo importante es el mayor riesgo de accidentabilidad detectado para las/-os enfermeras/-os en relación a los auxiliares de enfermería, independientemente del resto de variables incluidas en el modelo construido, lo cual nos ayuda a confirmar hallazgos univariantes anteriores de este estudio y de otros autores ${ }^{1,14}$.

Los trabajadores de la unidad de hemodiálisis, urgencias y laboratorios son los que, según este modelo, tienen mayor riesgo de accidentarse (magnitud de las ORs más importante). Del área de laboratorios cabe destacar cl que haya sido captada por el modelo, mientras que mostraba un menor riesgo en el estudio univariante. Otros autores, como R.D. McCormick y G. Maki, ya han comunicado este importante riesgo para los trabajadores de laboratorio, si bien en un estudio con análisis crudo ${ }^{2}$. Dichas áreas con mayor riesgo de accidentabilidad son candidatas a ser objeto de medidas preventivas colectivas de educación sanitaria y de una mayor vigilancia con el fin de minimizar la ocurrencia de aquellos factores que se relacionen con dicho riesgo.

También se ha detectado un riesgo de accidentabilidad mayor para los trabajadores con un tipo de contrato eventual, controlando por el resto de variables consideradas. Ésto puede explicarse por la mayor inexperiencia que suelen tener las personas con estos contratos, ya que, a menudo, están iniciando su carrera profesional. Una diferencia importante entre los sujetos con contrato fijo y aquéllos con contrato eventual es el hecho de que estos últimos no suelen trabajar con una continuidad suficiente para adquirir una práctica adecuada en el desarrollo de su actividad profesional. Por tanto, estas personas se beneficiarían de medidas preventivas co- 
lectivas como educación sanitaria específica para este tipo de riesgo biológico.

El riesgo de accidentarse para aquellas personas que habían notificado al menos un accidente en los 4 años previos al período de estudio, fue mayor que para las que no lo habían hecho, independientemente del resto de las variables del modelo. La explicación de este fenómeno es compleja, ya que esto puede deberse a una conducta de mayor accidentabilidad de algunas personas, a una conducta de mayor notificación o a un posible factor de confusión no tenido en cuenta, como es el área de trabajo en que se encontraba ese trabajador cuando se accidentó, ya que es posible que ese accidente previo lo sufriera mientras trabajaba en un área de mayor riesgo de accidentabilidad que en la que se encontraba trabajando en el momento de realizar este estudio. En cualquier caso, la sugerencia de la posible existencia de una conducta de mayor accidentabilidad es suficientemente importante para valorarlo en futuras investigaciones.

La construcción de un modelo que permita el cálculo de la probabilidad individual de accidentarse posibilita la selección de los trabajadores del Hospital sobre los que la implantación de medidas preventivas supondría un mayor beneficio. Además, es un hallazgo útil para la elaboración de estudios posteriores. Por ejemplo, si se decidiera realizar un estudio experimental para comprobar el beneficio que reportaría la utilización de un nuevo instrumental o una intervención preventiva en la disminución de estos accidentes, se necesitarían dos grupos de comparación en los que la probabilidad de accidentarse fuera similar. Una vez calculada dicha probabilidad con cste modelo, se podrían formar dos grupos homogéneos para realizar comparaciones ajustadas por el riesgo de accidentarse.

La información aquí recogida es de gran utilidad para determinar características relacionadas con un mayor riesgo de accidentabilidad y, por tanto, de gran valía para la implantación de medidas preventivas de estos accidentes, permitiendo dirigirlas hacia aquel personal sobre el que reportarían un mayor beneficio por ser de más riesgo.

\section{AGRADECIMIENTOS}

A Alberto Fernández y Emilio Perea-Milla de la Escuela Andaluza de Salud Pública, por las importantes sugerencias que aportaron para la realización de este trabajo.

\section{BIBLIOGRAFÍA}

1. Comisión Central de Salud Laboral del INSALUD. Accidentes biológicos en profesionales sanitarios. Madrid: International Marketing and Communications; 1995.

2. McCormick RD, Maki DG. Epidemiology of needle-stick injuries in hospital personnel. Am J Med 1981; 70: 928-932.

3. Centers for Desease Control. Update: Provisional Public Health Service recommendations for chemoprophylaxis after occupational exposure to HIV. MMWR 1996; 22(45): 468-472.

4. Jagger J, Hunt EH, Brand-Elnaggar J, Pearson RD. Rates of needle-stick injury caused by various devices in a university hospital. N Engl J Med 1988; 319: 284-288.

5. Fitch KM, Pérez L, de Andrés R, Nájera R. Occupational transmission of HIV in health care workers. Eur J Pub Health 1995; 5: 175-186.

6. De Andrés R, Pérez L, Contreras G, Nájera R, and the European Collaborative Study Group on Accidental Exposure to HIV. Update on European Union multicenter study of occupational exposure to HIV in health care workers. En: XI International Conference on AIDS; 1996 Jul; Vancouver.

7. Mitsui T, Iwano K, Masuko K, Yamazaki C, Okamoto $\mathrm{H}$, Tsuda $\mathrm{F}$ et al. Hepatitis $\mathrm{C}$ virus infection in medical personnel after needlestick accident. Hepatology 1992; 16: 1109-1114.

8. Alter MJ, The detection, transmission, and outcome of hepatitis $C$ virus infection. Infectious Agents Dis 1993; 2(3): 155-166.

9. The Association for Practitioners in Infection Control. APIC position paper: Prevention of device-mediated blood-borne infections to health care workers. Am J Infect Control 1993; 21: 76-78. 
10. Mercier C. Reducing the incidence of sharps injuries. Br J Nurs 1994; 3(17): 897-901.

11. Jagger J. EPINet: Exposure Prevention Information Network. Virginia: Becton Dickinson; 1992.

12. Centers for Desease Control, World Health Organization. Epilnfo 6, versión 6.02. 1994.

13. Hosmer DW, Lemeshow SA. Applied logistic regression. New York: John Wiley; 1989.

14. Whitby M, Stead P, Najman JM. Needlestick injuries: impact of a recapping device and an associated education program. Infect Control Hosp Epidemiol 1991; 12: 220-225.

15. Ippolito G, de Carli G, Puro V, Petrosillo N, Arici $C$, Bertucci $R$ et al. Device-specific risk of needlestick injury in italian health care workers. JAMA 1994; 272: 607-610.

16. Jagger J, Hunt EH, Pearson RD. Estimated cost of needlestick injuries for six major needle devices. Infect Control Hosp Epidemiol 1990; 11: 584-588.
17. Centers for Desease Control. Guidelines for prevention of transmission of human immunodeficiency virus and hepatitis $B$ virus to health-care and public-safety workers. MMWR 1989; 38 : S-6.

18. Goetz A, Yu CM, Muder RR. Entering first-year residents' experiences and knowledge of infection control of hepatitis B and HIV, at five universityaffiliated hospitals. Acad Med 1992; 67(4): 275 276.

19. Buss PW, McCabe M, Verrier ER. Attitudes of paediatricians to HIV and hepatitis B virus infection. Arch Dis Child 1991; 66(8): 961-964.

20. Williams S, Gooch C, Cockcroft A. Hepatitis B immunization and exposure to blood among surgical staff. Br J Surg 1993; 80: 714-716.

21. Rattner SL, Norman SA, Berlin JA. Percutaneous injuries on the «front line»: a survey of housestaff and nurses. Am J Prev Med 1994; 10: 372-377. 